05

\title{
Особенности фокусировки спиновых волн в кристаллах EuO и EuS
}

\author{
(C) С.М. Бахарев ${ }^{1,2}$, С.П. Савченко ${ }^{1}$, А.П. Танкеев ${ }^{1}$ \\ ${ }^{1}$ Институт физики металлов им. М.Н. Михеева УрО РАН, \\ Екатеринбург, Россия \\ ${ }^{2}$ Уральский фредеральный университет им. Б.Н. Ельцина, \\ Екатеринбург, Россия \\ E-mail: bakharevsm@imp.uran.ru
}

(Поступила в Редакцию 13 августа 2018 г.)

Исследованы особенности фокусировки спиновых волн в кристаллах $\mathrm{EuO}$ и $\mathrm{EuS}$. Показано, что в длинноволновом приближении фокусировка спиновых волн отсутствует: она наблюдается лишь для волновых векторов во второй половине зоны Бриллюэна $(a q \gtrsim \pi$, где $a-$ постоянная решетки, $q-$ величина волнового вектора). Обнаружено, что для кристаллов $\mathrm{EuO}$ и $\mathrm{EuS}$ направления фокусировки спиновых волн отличаются вследствие разных знаков обменных интегралов, отвечающих за обменное взаимодействие магнитного момента со вторыми соседями. Определены направления, вдоль которых формируется каустика магнонов.

Работа выполнена в рамках государственного задания по теме „Спин“ АААА-А18-118020290104-2 и проекту № 32-1.1.3.5 Программы фундаментальных исследований Президиума РАН согласно контракту Минобрнауки № 14.Z50.31.0025, а также гранта РФФИ мол_а (проект № 18-32-00139).

DOI: $10.21883 /$ FTT.2019.02.47123.205

\section{1. Введение}

В настоящей работе рассмотрим особенности фокусировки спиновых волн в кристаллах $\mathrm{EuO}$ и EuS. Как и другие халькогениды европия (моноселенид $\mathrm{EuSe}$, монотеллурид ЕuТе и др.), указанные соединения классифицируются как магнитные полупроводники, главная особенность которых состоит в сильном взаимодействии их намагниченности и электронов проводимости. Отсюда следует возможность управления воздействием на магнитную подсистему электронной спиновой системой. Это обстоятельство открыло перспективы широкого практического применения магнитных полупроводников в современной электронике [1-5].

Заметим, что характер магнитного упорядочения в указанных выше проводниках определяется линейными размерами входящих в их формулу анионов: EuTe антиферромагнетик, EuSe обнаруживает как антиферромагнитное, так и ферромагнитное упорядочение в зависимости от выбранного интервала температур, а EuO и $\mathrm{EuS}$ являются ферромагнетиками. По существу различие в свойствах соединений, входящих в эту группу вызвано только размерами анионов $\mathrm{EuTe}$ и $\mathrm{EuO}$ (от большого радиуса аниона $\mathrm{Te}^{2-}$, к малому радиусу аниона $\left.\mathrm{O}^{2-}\right)[6,7]$. Это заключение следует из экспериментальных данных по измерению обменных констант, полученных в [6,7], где было показано, что обменные связи ионов $\mathrm{Eu}^{2+}$ в соединениях $\mathrm{EuO}, \mathrm{EuS}$ и EuTe меняются гладко и непрерывно с изменением расстояния между ионами. Обменное взаимодействие ближайших соседей ферромагнитно на наибольших расстояниях (EuTe) и увеличи- вается с уменьшением межионного расстояния. Обмен со вторыми соседями является антиферромагнитным на самых больших расстояниях. Он уменьшается по мере уменьшения межионного расстояния и, наконец, согласно измерениям авторов статей $[6,7]$ (и в противоречие с более ранними результатами [8] становится ферромагнитным на малых расстояниях (EuO).

Электронный спектр магнитных полупроводников определяется двумя подсистемами - подвижными носителями заряда (электронами проводимости и дырками) и более локализованными электронами атомов переходных или редкоземельных металлов, содержащих недостроенные $d$ - или $f$-оболочки. Магнитные полупроводники характеризуются, как правило, наличием косвенного обменного взаимодействия между этими $d$ или $f$-ионами. В решетке магнитного полупроводника магнитные $d$ - или $f$-ионы разделены немагнитными, и поэтому их волновые функции не перекрываются. Прямое обменное взаимодействие между ними отсутствует. Однако при этом возникает косвенное взаимодействие, обусловленное тем, что волновые функции перекрываются через волновые функции немагнитных ионов. Кроме косвенного обменного взаимодействия между парамагнитными ионами через неподвижные немагнитные ионы, в магнитных полупроводниках может иметь место непрямое обменное взаимодействие через подвижные носители заряда. С магнитной точки зрения, считается, что выделенная группа соединений представляет собой систему магнитных моментов, связанных обменными взаимодействиями, которые хорошо моделируются изотропными гейзенберговскими гамильтонианами. 
Наша задача состоит в исследовании особенностей распространения спиновых волн в магнитных полупроводниках на примере двух базовых соединений $\mathrm{EuO}$ и $\mathrm{EuS}$, имеющих структуру каменной соли (ГЦК). Заметим, что ионы $\mathrm{Eu}^{2+}$ в соединениях $\mathrm{EuO}$ и $\mathrm{EuS}$ находятся в состоянии с ${ }^{8} S_{7 / 2}$, когда орбитальный момент электронов отсутствует $(L=0, S=J=7 / 2)$. По этой причине указанные соединения имеют малую кристаллографическую магнитную анизотропию: поле анизотропии для $\mathrm{EuO}$ равно $190 \mathrm{Oe}$, а для EuS меньше 30 Ое. Заметим, что обменное взаимодействие существенно только внутри двух первых координационных сфер (число соседей $z_{1}=12, z_{2}=6$ соответственно) [2].

Для каждого типа решетки необходимо, во-первых, определить интервал частот, в котором реализуются условия фокусировки спиновых волн. Во-вторых, найти направления в кристалле и необходимые условия для формирования каустики - огибающей семейства магнонов, не сходящихся в одной точке, вблизи которой резко возрастает интенсивность магнонного поля. Необходимо также отследить изменение условий фокусировки магнонов при изменении величины и знака параметра обменного взаимодействия (обменного интеграла) со вторыми соседями.

В рамках феноменологического подхода, когда динамика магнитного момента описывается с помощью уравнений движения без затухания при условии сохранения величины магнитного момента, нами будет рассчитан спектр магнонов для гранецентрированной кубической (ГЦК) решетки в двух случаях: 1) при учете обменного взаимодействия только с ближайшими соседями; 2) с учетом обменного взаимодействия с ближайшими и следующими за ними соседями.

Поскольку групповая скорость магнона перпендикулярна изоэнергетической поверхности, то, зная закон дисперсии спиновых волн, можно определить направления фокусировки и дефокусировки магнонов. Для количественного описания указанных явлений нами будут использованы два подхода, ранее разработанные для фононов: 1) метод, основанный на вычислении коэффициента усиления потока частиц, и 2) метод, основанный на вычислении углового распределения плотности состояний.

Первый из упомянутых методов был разработан Марисом [9] в 1971 году и позднее развит в работах [10-16] для количественной оценки фокусировки фононов в кубических кристаллах. Коэффициент усиления потока фононов определяется как отношение потока тепла данной поляризации в исследуемом кристалле к соответствующему потоку тепла в изотропной среде для выбранного направления волнового вектора. Этот коэффициент показывает, во сколько раз изменится интенсивность потока данной колебательной моды по сравнению с изотропной средой. Коэффициент усиления позволяет рассчитать локализацию параболических линий и каустик. На этих линиях кривизна изоэнергетической поверхности обращается в нуль, и, поскольку коэффициент усиления обратно пропорционален кривизне (он имеет сингулярность), последнее обстоятельство указывает на каустику.

Второй метод предложен в работах [17-19] для вычисления угловых распределений плотности фононных состояний (ПФС) квазипоперечных фононных мод. Он основан на анализе структуры поверхности постоянной энергии поперечных мод при наличии вогнутых участков на указанной поверхности и расчете углов, характеризующих фокусировку фононов. Плотность состояний определяется отношением сектора углов групповых скоростей, распространяющихся в изотропной среде, к сектору углов групповых скоростей фононов, распространяющихся в рассматриваемом кристалле. В области фокусировки ПФС больше единицы, в области дефокусировки - меньше единицы. Этот подход предполагается использовать для количественной оценки плотности состояний магнонов при наличии вогнутых областей на изоэнергетической поверхности.

\section{2. Спектр спиновых волн в ферромагнетиках с ГЦК-решеткой}

В этом разделе приведены результаты расчета спектра спиновых волн для ферромагнетиков с ГЦК-решеткой во всей зоне Бриллюэна. В качестве примера рассмотрены соединения $\mathrm{EuO}$ и $\mathrm{EuS} \mathrm{c} \mathrm{кристаллической} \mathrm{структурой}$ типа каменной соли $[6,7,20]$. Ограничимся рассмотрением области низких температур - ниже $4.2 \mathrm{~K}$. Как показано в работах [6,7], при этих температурах постоянные обменного взаимодействия слабо зависят от температуры. Заметим, что магнитное упорядочение ионов $\mathrm{Eu}^{2+}$ в сферически-симметричных спиновых состояниях

${ }^{8} S_{7 / 2}$, расположенных в решетках с высокой симметрией, является следствием изотропного обменного взаимодействия с ближайшими ( $n n)$ и следующими ( $n n n)$ соседями, которое можно записать в виде Гейзенберга $[6,7]$

$$
\mathscr{H}_{\text {exch }}=-J_{1} \sum_{n n} \mathbf{S}_{0} \mathbf{S}_{n n}-J_{2} \sum_{n n n} \mathbf{S}_{0} \mathbf{S}_{n n n}
$$

где $J_{1}$ и $J_{2}$ - постоянные обменного взаимодействия для ближайших и следующих за ними соседей; $\mathbf{S}_{0}, \mathbf{S}_{n n}$ и $\mathbf{S}_{n n n}-$ спины в начальной, в $n n$ и $n n n$ позициях соответственно. Для этого гамильтониана легко получаем энергию спиновых волн

$$
\hbar \omega(\mathbf{q})=2 S\left\{J_{1} \sum_{n n}\left(1-e^{-i \mathbf{q} \mathbf{r}_{n n}}\right)+J_{2} \sum_{n n n}\left(1-e^{-i \mathbf{q} \mathbf{r}_{n n n}}\right)\right\} .
$$

В ГЦК-решетке количество ближайших соседей (nn) 12 штук, расположенных в центре грани куба; количество следующих за ближайшими соседями (nnn) 6 штук, расположенных по ребрам куба. Введем величины $\omega_{E}=2 S J_{1} / \hbar$ и $\xi=J_{2} / J_{1}$ и представим спектр (2) 
в виде

$$
\begin{aligned}
\omega(\mathbf{q})= & 2 \omega_{E}\left[2 \left(3-\cos \left(\frac{a}{2} q_{x}\right) \cos \left(\frac{a}{2} q_{z}\right)\right.\right. \\
- & \left.\cos \left(\frac{a}{2} q_{y}\right) \cos \left(\frac{a}{2} q_{z}\right)-\cos \left(\frac{a}{2} q_{x}\right) \cos \left(\frac{a}{2} q_{y}\right)\right) \\
& \left.+\xi\left(3-\cos \left(q_{x} a\right)-\cos \left(q_{y} a\right)-\cos \left(q_{z} a\right)\right)\right]
\end{aligned}
$$

где $a-$ постоянная решетки. В длинноволновом приближении $(a q \ll 1)$ выражение $(3)$ преобразуется к виду

$$
\omega(q)=\omega_{E}(1+\xi)(a q)^{2} .
$$

Из формулы (4) следует, что имеется ограничение на выбор параметра обменного взаимодействия со вторыми соседями: $|\xi|<1$. В противном случае необходимо учитывать взаимодействие с тремя и более соседями.

В работах [6,7] авторы методом неупругого рассеяния нейтронов получили спектр спиновых волн в поликристаллах $\mathrm{EuO}$ и $\mathrm{EuS}$ во всей зоне Бриллюэна. Для параметров обменного взаимодействия спинов $J_{1}$ и $J_{2}$ были получены следующие значения: у $\mathrm{EuO} \quad J_{1} / k_{\mathrm{B}}=0.606 \pm 0.008 \mathrm{~K} \quad$ и $J_{2} / k_{\mathrm{B}}=0.119 \pm 0.015 \mathrm{~K}$; y $\operatorname{EuS} J_{1} / k_{\mathrm{B}}=0.236 \pm 0.009 \mathrm{~K}$ и $J_{2} / k_{\mathrm{B}}=-0.118 \pm 0.011 \mathrm{~K}\left(k_{\mathrm{B}}\right.$ - постоянная Больцмана). Очевидно, что для кристалла $\mathrm{EuO}$ параметр $J_{2}>0$, а для $\operatorname{EuS} J_{2}<0$.

Заметим, что, обмен между $(n n)$ ионами $\mathrm{Eu}^{2+}$ является косвенным из-за обычно незанятых $5 d$ уровней [8]. Следующие соседи (nnn) экранированы анионами, поэтому обменная связь с (nnn) ионами $\mathrm{Eu}^{2+}$ включает в себя несколько механизмов. Одним из них является суперобмен, в котором участвует $p$-электрон аниона, а другим - формирование пар $(n n n) \mathrm{Eu}^{3+}-\mathrm{Eu}^{+}$для передачи $4 f$-электрона.

Поверхность зоны Бриллюэна ГЦК-решетки имеет вид усеченного октаэдра, уравнение которой можно представить в виде

$q_{\max }(\theta, \varphi)=\frac{2 \pi}{a} \min \left\{\frac{1}{\left|n_{x}\right|}, \frac{1}{\left|n_{y}\right|}, \frac{1}{\left|n_{z}\right|}, \frac{3 / 2}{\left|n_{x}\right|+\left|n_{y}\right|+\left|n_{z}\right|}\right\}$,

где $n_{i}$ - компоненты единичного волнового вектора.

Спектр спиновых волн (3), в ГЦК-решетке определяется тремя параметрами: $\omega_{E}$, $\xi$ и $a$. Исследуем зависимость этого спектра в терминах приведенной частоты $\omega / \omega_{E}$ от безразмерного волнового вектора $a q$. При этом спектр будет определяться одним безразмерным параметром $\xi$, а также углами $\theta$ и $\varphi$, задающими направление волнового вектора q. В большинстве случаев функция $\omega(a q) / \omega_{E}$ монотонно возрастает как для положительных, так и для отрицательных значений параметра $\xi$ в симметричных направлениях волнового вектора q. Исключение составляет ситуация $\xi=0.8$ для направления [110], когда $\omega(a q) / \omega_{E}$ имеет экстремум во второй половине зоны Бриллюэна при $a q \approx 5.6$. Это указывает, что есть такая область в спектре, где в кристалле с одной и той же энергией могут распространяться два потока магнонов.

\section{3. Групповая скорость и коэффициент усиления потока магнонов}

В этом разделе запишем выражение для групповой скорости магнонов, определим коэффициент усиления потока магнонов и получим условие для формирования каустик спиновых волн.

\section{1. Групповая скорость магнонов}

Групповая скорость магнонов определяется следующим соотношением:

$$
\mathbf{V}(\mathbf{q})=\frac{\partial \omega(\mathbf{q})}{\partial \mathbf{q}}=V_{n} \mathbf{n}+V_{\theta} \mathbf{e}_{\theta}+V_{\varphi} \mathbf{e}_{\varphi},
$$

где

$$
V_{n}=\frac{\partial \omega}{\partial q}, \quad V_{\theta}=\frac{1}{q} \frac{\partial \omega}{\partial \theta}, \quad V_{\varphi}=\frac{1}{q \sin \theta} \frac{\partial \omega}{\partial \varphi},
$$

а векторы

$$
\begin{aligned}
& \mathbf{n}=\mathbf{q} / q=(\sin \theta \cos \varphi, \sin \theta \sin \varphi, \cos \theta) \\
& \mathbf{e}_{\theta}=(\cos \theta \cos \varphi, \cos \theta \sin \varphi,-\sin \theta) \\
& \mathbf{e}_{\varphi}=(-\sin \varphi, \cos \varphi, 0)
\end{aligned}
$$

образуют тройку взаимно ортогональных векторов. Углы $\theta$ и $\varphi$ задают направление волнового вектора $\mathbf{q}$ (в системе координат по ребрам куба угол $\theta$ отсчитывается от оси $Z$, а угол $\varphi-$ от оси $X$ ). В симметричных направлениях [100], [110] и [111] угловые компоненты групповой скорости $V_{\theta}=V_{\varphi}=0$ : в этих направлениях групповая и фазовая скорости коллинеарны. Отметим, что в плоскости грани куба и диагональной плоскости угловая компонента $V_{\varphi}=0$ для любого угла $\theta$.

\section{2. Коэффициент усиления потока магнонов}

Для количественного описания эффекта фокусировки магнонов применим метод, предложенный Марисом в работе [9] для оценки фокусировки фононов. Автор воспользовался моделью изотропной среды как системой сравнения и ввел понятие коэффициента усиления потока фононов $A(\theta, \varphi)-$ „enhancement factor" ${ }^{6}$, который также известен [21], как ,amplification factor“. Согласно [9] коэффициент $A(\theta, \varphi)$ определяется отношением потока фононов данной поляризации для выбранного направления волнового вектора к соответствующему потоку в изотропной среде. Позже в работе [10] было показано, что коэффициент усиления характеризует изоэнергетическую поверхность будучи обратно пропорциональным ее гауссовой кривизне $A \sim 1 / K$.

По аналогии с работой [10] коэффициент усиления $A(\mathbf{q})$, характеризующий отличие потоков магнонов в 
анизотропном $(a q \gtrsim \pi)$ и изотропном $(a q \ll 1)$ случаях, может быть определен через гауссову кривизну $K(\mathbf{q})$ следующим образом:

$$
A(\mathbf{q})=\frac{V_{n}}{V q^{2}|K|} .
$$

Согласно [22], при заданной частоте спиновых волн $\omega$ полная (или гауссова) кривизна поверхности $\mathbf{q}(\theta, \varphi)$ определяется следующим выражением

$$
K(\mathbf{q})=\frac{L N-M^{2}}{E G-F^{2}},
$$

где коэффициенты $E, F$ и $G$ характеризуют первую квадратичную форму $d \mathbf{q}^{2}$, а коэффициенты $L, N$ и $M-$ вторую квадратичную форму $\left(-d \mathbf{q} d \mathbf{n}_{V}\right)$, где $\mathbf{n}_{V}=\mathbf{V} / V-$ единичный вектор групповой скорости магнона. Эти коэффициенты выражаются через частные производные волнового вектора $\mathbf{q}(\theta, \varphi)$ по углам $\theta$ и $\varphi[22]: E=\mathbf{q}_{\theta}^{2}$, $F=\mathbf{q}_{\theta} \mathbf{q}_{\varphi}, \quad G=\mathbf{q}_{\varphi}^{2} ; \quad L=\mathbf{q}_{\theta \theta} \mathbf{n}_{V}, M=\mathbf{q}_{\theta \varphi} \mathbf{n}_{V}, N=\mathbf{q}_{\varphi \varphi} \mathbf{n}_{V}$. После вычисления частных производных волнового вектора $\mathbf{q}(\theta, \varphi)$ по углам $\theta$ и $\varphi$ для знаменателя в выражении (10) получаем

$$
E G-F^{2}=q^{4} \sin ^{2} \theta\left(\frac{V}{V_{n}}\right)^{2}
$$

а для коэффициентов $L, M$ и $N$ имеем следующие соотношения:

$$
\begin{gathered}
L=\frac{q}{V V_{n}}\left[-q \frac{V_{\theta}^{2}}{V_{n}} \frac{\partial V_{n}}{\partial q}+2 V_{\theta}\left(\frac{\partial V_{n}}{\partial \theta}-V_{\theta}\right)-V_{n}\left(\frac{\partial V_{\theta}}{\partial \theta}+V_{n}\right)\right], \\
M=\frac{q}{V V_{n}}\left[-q \sin \theta \frac{V_{\theta} V_{\varphi}}{V_{n}} \frac{\partial V_{n}}{\partial q}+V_{\theta} \frac{\partial V_{n}}{\partial \varphi}-V_{n} \frac{\partial V_{\theta}}{\partial \varphi}\right. \\
\left.+V_{\varphi}\left(\frac{\partial V_{n}}{\partial \theta} \sin \theta-2 V_{\theta} \sin \theta+V_{n} \cos \theta\right)\right], \\
N=\frac{q}{V V_{n}} \sin \theta\left[-q \frac{V_{\varphi}^{2}}{V_{n}} \sin \theta \frac{\partial V_{n}}{\partial q}-V_{n}\left(\frac{\partial V_{\varphi}}{\partial \varphi}+V_{n} \sin \theta\right.\right. \\
\left.\left.+V_{\theta} \cos \theta\right)+2 V_{\varphi}\left(\frac{\partial V_{n}}{\partial \varphi}-V_{\varphi} \sin \theta\right)\right] .
\end{gathered}
$$

Особый интерес представляют точки (или линии), в которых кривизна изоэнергетической поверхности обращается в нуль. В этих точках коэффициент усиления имеет сингулярность, указывающую на явление каустики магнонов. Каустика - это особые линии (в двухмерном случае) и особые поверхности, вблизи которых резко возрастает интенсивность спин-волнового поля [10,23]. Кривизна регулярной поверхности (дважды непрерывно дифференцируемой) обращается в нуль в параболических точках. Это такие точки на поверхности, в которых соприкасающийся параболоид вырождается в параболический цилиндр. Из выражения для кривизны (10) при заданной частоте спиновых волн $\omega$ получаем условие для определения параболических точек: $L N=M^{2}$. Поскольку в этих точках кривизна поверхности обращается в нуль, а коэффициент усиления обратно пропорционален $K$, то выше мы получили условия для нахождения точек (или линий) каустик, в которых интенсивность магноного поля резко возрастает. В изотропном случае $(a q \ll 1)$ коэффициент усиления $A=1$.

\section{4. Фокусировка и анизотропия плотности магнонных состояний}

Рассмотрим фокусировку спиновых волн в исследуемых кристаллах. Спектр магнонов определяется выражением (3), в котором учитывается только обменное взаимодействие магнитных моментов. Величина $\xi$ является варьируемым параметром. Как следует из формулы (4), в длинноволновом приближении спектр магнонов изотропен - он не зависит от направления волнового вектора. Поэтому в случае $a q \ll 1$ фокусировка спиновых волн отсутствует. Зависимость частоты магнонов от направления q возникает во второй половине зоны Бриллюэна $(a q \gtrsim \pi)$.

Определить направления фокусировки и дефокусировки магнонов можно из структуры изоэнергетических поверхностей, поскольку групповая скорость перпендикулярна этой поверхности в заданной точке и определяет поток магнонов. В качестве примера рассмотрим магнитные полупроводники $\mathrm{EuO}$ и $\mathrm{EuS} \mathrm{c} \mathrm{параметрами}$ обменного взаимодействия магнитного момента со вторыми соседями $\xi=J_{2} / J_{1}=0.2$ и -0.5 , соответственно (согласно работам $[6,7]$ ).

По аналогии с известным анализом фокусировки фононов [17-19], для магнонов введем углы $\theta_{0}, \theta_{1}, \theta_{2}$ и $\theta_{V 0}$, характеризующие фокусировку спиновых волн при наличии вогнутых участков на изоэнергетической поверхности (схема и иллюстрация расчета этих углов подробно описана в работах [17-19]). Эти углы будем отсчитывать от направления фокусировки $\theta=\theta_{F}$. Угол $\theta_{1}$ определяет направление волнового вектора, для которого вектор групповой скорости $\mathbf{V}$ параллелен направлению фокусировки. Углы $\theta_{V 0}$ определяют направления групповых скоростей в точках нулевой кривизны на изоэнергетической поверхности. В этих точках вектор групповой скорости магнона имеет максимальное схождение к направлению фокусировки, и этот угол мы обозначим, как $\theta_{V 0}=\theta_{F}-\theta_{V}\left(\theta_{0}\right)$. Углы $\pm \theta_{0}$ задают направления волновых векторов к точкам нулевой кривизны. Очевидно, что величину сектора волновых векторов, соответствующих области фокусировки, будут определять групповые скорости $\mathbf{V}\left( \pm \theta_{2}\right)$, коллинеарные направлениям $\mathbf{V}$ в точках нулевой кривизны, а именно $\theta_{F}-\theta_{2} \leqslant \theta \leqslant \theta_{F}+\theta_{2}$. Для нахождения характерных углов $\theta_{i}$ и $\theta_{V 0}$ определим направление групповой скорости $\theta_{V}(\mathbf{q})$ через угловые координаты волнового вектора $\theta$ и $\varphi$. Из условия $\mathbf{V q}=V q \cos (\alpha(\mathbf{q}))$ 
Таблица 1. Характеристические углы $\theta_{i[J]}^{\{I\}}$ и плотность магнонных состояний для направления $[J]$ в плоскости $\{I\}$ (индексы $[J]$ и $\{I\}$ указаны во втором столбце) для различных частот спиновых волн $\omega / \omega_{E}$ и параметров обменного взаимодействия со вторыми соседями $\xi=J_{2} / J_{1}$

\begin{tabular}{c|c|c|c|c|c|c|c|c}
\hline$\xi$ & $\omega / \omega_{E},[J]\{I\}$ & $\theta_{0[J]}^{\{I\}}$ & $\theta_{1[J]}^{\{I\}}$ & $\theta_{2[J]}^{\{I\}}$ & $\theta_{V 0[]]}^{\{I\}}$ & $n_{F[[]]}^{\{I\}}$ & $n_{D I[]]}^{\{I\}}$ & $n_{F D[J]}^{\{I\}}$ \\
\hline 0.8 & $15.9[110]\{100\}$ & $37.5^{\circ}$ & $42.3^{\circ}$ & $43^{\circ}$ & $8.0^{\circ}$ & 5.4 & 0.05 & 99.1 \\
\hline-0.5 & $5.9[110]\{110\}$ & $26.4^{\circ}$ & $33.1^{\circ}$ & $34.2^{\circ}$ & $14.0^{\circ}$ & 2.4 & 0.74 & 3.3 \\
\hline-0.8 & $1.0[110]\{110\}$ & $13.1^{\circ}$ & $21.4^{\circ}$ & $23.8^{\circ}$ & $2.2^{\circ}$ & 10.8 & 0.75 & 14.3 \\
\hline-0.8 & $2.3[100]\{100\}$ & $15.3^{\circ}$ & $25.0^{\circ}$ & $28.4^{\circ}$ & $2.2^{\circ}$ & 12.5 & 0.40 & 31.6 \\
\hline-0.8 & $2.3[100]\{110\}$ & $45.6^{\circ}$ & $52.4^{\circ}$ & $53.3^{\circ}$ & $9.8^{\circ}$ & 5.4 & 0.46 & 11.9 \\
\hline-0.8 & $2.3[110]\{110\}$ & $28.9^{\circ}$ & $34.4^{\circ}$ & $35.1^{\circ}$ & $26.4^{\circ}$ & 1.3 & 0.87 & 1.53
\end{tabular}

определим угол между групповой скоростью и волновым вектором: $\alpha(\mathbf{q})= \pm \arccos \left(V_{n}(\mathbf{q}) / V(\mathbf{q})\right)$.

Рассмотрим случаи волновых векторов, лежащих в плоскостях $\{100\}$ и $\{110\}$, для которых углы $\varphi=0$ и $\pi / 4$, а компонента групповой скорости $V_{\varphi}=0$. Тогда $\alpha(\mathbf{q})=\arctan \left(V_{\theta}(\mathbf{q}) / V_{n}(\mathbf{q})\right)$. В системе координат с осями по ребрам куба угол между осью $Z$ и направлением групповой скорости равен

$$
\theta_{V}(\mathbf{q})=\theta+\alpha(\mathbf{q})=\theta+\arctan \left(\frac{V_{\theta}(\mathbf{q})}{V_{n}(\mathbf{q})}\right) .
$$

Из последнего равенства следует, что знак компоненты $V_{\theta}$ определяет, в какую сторону будет отклоняться вектор групповой скорости относительно волнового вектора магнона. Поскольку в изотропном случае $(a q \ll 1)$ направления фазовой и групповой скоростей магнона совпадают с направлением волнового вектора, то мы получаем возможность рассматривать изотропный случай как систему сравнения при анализе влияния фокусировки магнонов на изменение плотности магнонных состояний в случае анизотропного спектра. Угол $\theta_{1}$ определяется из условия $\theta_{V}=\theta_{F}$ и $\theta=\theta_{F}-\theta_{1}$

$$
\theta_{1}=\arctan \left(\frac{V_{\theta}\left(\omega, \theta_{F}-\theta_{1}\right)}{V_{n}\left(\omega, \theta_{F}-\theta_{1}\right)}\right) .
$$

Для нахождения углов $\theta_{0}$ и $\theta_{V 0}$ необходимо построить функцию $\theta_{V}(\omega, \theta)$ при заданной частоте $v=\omega / 2 \pi$ и найти ее минимум. Положение минимума $d \theta_{V} /\left.d \theta\right|_{\theta=\theta_{F}-\theta_{0}}$ определяет точку нулевой кривизны с углом $\theta_{0}$, а значение функции $\theta_{V}\left(\omega, \theta_{0}\right)$ дает величину угла $\theta_{V 0}$

$$
\theta_{V 0}=\theta_{0}-\arctan \left(\frac{V_{\theta}\left(\omega, \theta_{F}-\theta_{0}\right)}{V_{n}\left(\omega, \theta_{F}-\theta_{0}\right)}\right) .
$$

После построения вектора групповых скоростей $\mathbf{V}\left( \pm \theta_{\mathbf{0}}\right)$ определим углы „схождения“ групповых скоростей $\pm \theta_{V 0}$. Угол $2 \theta_{V 0}$ определяет область фокусировки магнонов. Очевидно, что величину сектора волновых векторов, соответствующих фокусировке магнонов, будут определять групповые скорости $\mathbf{V}\left( \pm \theta_{2}\right)$, коллинеарные направлениям $\mathbf{V}$ в точках нулевой кривизны, а именно $\theta_{F}-\theta_{2} \leqslant \theta \leqslant \theta_{F}+\theta_{2}$. Уравнение для определения угла $\theta_{2}$ имеет вид

$$
\theta_{V 0}=\theta_{2}-\arctan \left(\frac{V_{\theta}\left(\omega, \theta_{F}-\theta_{2}\right)}{V_{n}\left(\omega, \theta_{F}-\theta_{2}\right)}\right)
$$

Таким образом, расходящийся сектор волновых векторов $\theta_{F}-\theta_{2} \leqslant \theta \leqslant \theta_{F}+\theta_{2}$ превращается в сходящийся к направлению фокусировки сектору групповых скоростей $\theta_{F}-\theta_{V 0} \leqslant \theta_{V} \leqslant \theta_{F}+\theta_{V 0}$.

По аналогии с анализом фокусировки фононов $[18,19]$, для магнонов введем среднюю плотность состояний, приходящуюся на единичный угол для областей фокусировки $\theta_{F}-\theta_{V 0} \leqslant \theta_{V} \leqslant \theta_{F}+\theta_{V 0}$ и изотропной среды $\theta_{F}-\theta_{2} \leqslant \theta \leqslant \theta_{F}+\theta_{2}$. Поскольку расходящийся сектор волновых векторов с углом $2 \theta_{2}$ превращается в сходящийся сектор векторов групповых скоростей фононов с углом $2 \theta_{V 0}$, то средняя плотность состояний в областях фокусировки магнонов будет больше, чем в изотропной среде в отношении:

$$
n_{F I}=\frac{N_{F}}{N_{\text {Iso }}}=\frac{\theta_{2}}{\theta_{V 0}} .
$$

Для области дефокусировки средняя плотность состояний $n_{D I}$ будет меньше, чем $N_{\text {Iso }}$ в отношении

$$
n_{D I}=\frac{N_{D}}{N_{\text {Iso }}}=\frac{\pi-4 \theta_{2}}{\pi-4 \theta_{V 0}}
$$

для плоскости грани куба и

$$
n_{D I}=\frac{\pi-2 \theta_{2}}{\pi-2 \theta_{V 0}}
$$

для диагональной плоскости. Из формул (17) и (18) получим отношения средних плотностей состояний для областей фокусировки и дефокусировки магнонов, которые характеризуют анизотропию плотности состояний: $n_{F D}=N_{F} / N_{D}=n_{F D} / n_{D I}$. Характеристические углы $\theta_{0}, \theta_{1}, \theta_{2}$ и $\theta_{V 0}$ и плотность состояний магнонов для ряда случаев приведены в табл. 1. 

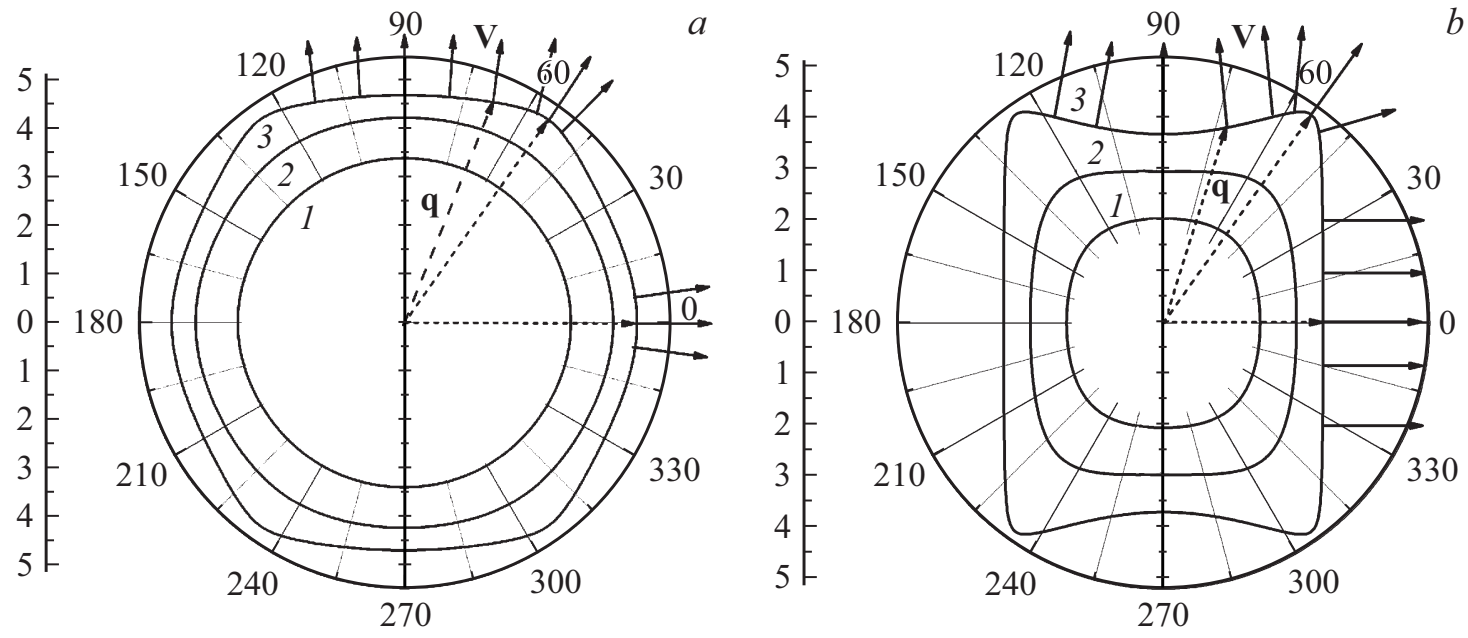

Рис. 1. Сечения поверхностей постоянной частоты $a q(\theta)$ в ГЦК решетке плоскостью $\{110\}$ в модели, учитывающей обменное

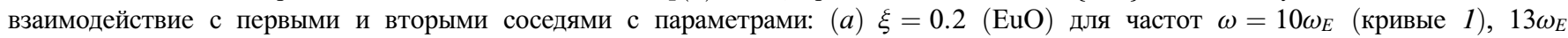
(кривые 2) и $14.3 \omega_{E}$ (кривые 3); (b) $\xi=-0.5$ (EuS) для частот $\omega=2 \omega_{E}$ (кривые 1 ), $4 \omega_{E}$ (кривые 2) и $5.9 \omega_{E}$ (кривые 3 ).

Отметим, что положения каустик спиновых волн в плоскости грани куба и диагональной плоскости задаются углами $\theta_{0}$ (в q-пространстве) и $\theta_{V 0}($ в $\mathbf{V}$-пространстве) и совпадают с положениями особенностей коэффициента усиления (расходимость).

Заметим, что этот метод разработан для конкретного сечения изоэнергетической поверхности $\varphi=$ const, поэтому компонента групповой скорости $V_{\varphi}$ и ее производные не учитываются. Кроме того он применим только в случаях, когда на изоэнергетической поверхности имеются вогнутые участки.

\section{1. Фокусировка и каустика спиновых волн в ГЦК решетке}

Анализ поверхностей постоянной энергии и расчет коэффициента усиления спиновых волн в ГЦК решетке показали, что с изменением величины параметра взаимодействия магнитного момента со вторыми соседями $\xi$ качественно меняется картина фокусировки. Так, при $\xi=0$ в направлении [100] имеет место локальная фокусировка спиновых волн, в направлении [110] фокусировка, а в [111] - дефокусировка. Увеличение $\xi$ до 0.2 (этот случай относится к кристаллу $\mathrm{EuO}$ ) приводит к тому, что в направлении [100] магноны дефокусируются, а в остальных направлениях картина фокусировки качественно не меняется (см. рис. 1,a). Однако для значений этого параметра $\xi$ спектр магнонов менее анизотропен, и поэтому фокусировка выражена слабее, чем для случая $\xi=0$ (см. табл. 2).

Дальнейшее увеличение параметра $\xi$ приводит к качественным изменениям анизотропии спектра и, соответственно, к изменению и условий фокусировки спиновых волн. На изоэнергетических поверхностях появляются вогнутые участки (см. рис. 2). Этот случай представляет особый интерес, поскольку в параболических точках (точки, в которых кривизна изоэнергетической поверхности обращается в нуль) коэффициент усиления имеет сингулярность $(A \rightarrow \infty)$. Эти точки указывают на каустику магнонов. При $\xi=0.8$ на частотах $\omega \approx 15.9 \omega_{E}$ в окрестности направлений [110] и [111] магноны фокусируются с формированием каустики (см. рис. 3, кривые 3 и 4). Углы $\theta_{0}$ и $\theta_{V 0}$, определяющие положения каустик, представлены в табл. 1. В направлении [100] происходит дефокусировка (см. рис. 2 и 3).

Отдельно необходимо отметить случай $\xi=0.8$, когда в диагональной плоскости при $\theta=\theta_{F}=12.3^{\circ}$ фокусируются магноны, и вблизи направления [111] $\left(\theta_{V}=52.7^{\circ}\right)$ происходит формирование каустики на частоте $\omega=15.9 \omega_{E}$ (см. рис. 2 и 3 ). Однако этот случай не отражен в табл. 1, поскольку относительно направления

Таблица 2. Значения коэффициентов усиления магнонов в симметричных направлениях для различных частот спиновых волн $\omega / \omega_{E}$ и параметров обменного взаимодействия со вторыми соседями $\xi$

\begin{tabular}{c|c|c|c|c}
\hline$\xi$ & $\omega / \omega_{E}$ & $A_{[100]}$ & $A_{[110]}$ & $A_{[111]}$ \\
\hline \multirow{2}{*}{0.8} & 10 & 0.32 & 1.53 & 2.10 \\
& 15.9 & $1.2 \cdot 10^{-3}$ & 3.76 & 9.16 \\
\hline \multirow{2}{*}{0.2} & 13 & 0.66 & 1.46 & 0.54 \\
& 14.3 & 0.52 & 2.47 & 0.05 \\
\hline \multirow{2}{*}{0} & 10 & 2.00 & 1.43 & 0.31 \\
& 11.9 & 2.68 & 16.77 & 0.015 \\
\hline \multirow{2}{*}{0.5} & 4 & 22.97 & $\infty$ & 0.09 \\
& 5.9 & $1.03 \cdot 10^{4}$ & 0.54 & $2 \cdot 10^{-3}$ \\
\hline \multirow{2}{*}{0.8} & 1 & 520.3 & 1.32 & 0.046 \\
& 2.3 & 22.14 & 0.23 & $5.5 \cdot 10^{-4}$
\end{tabular}




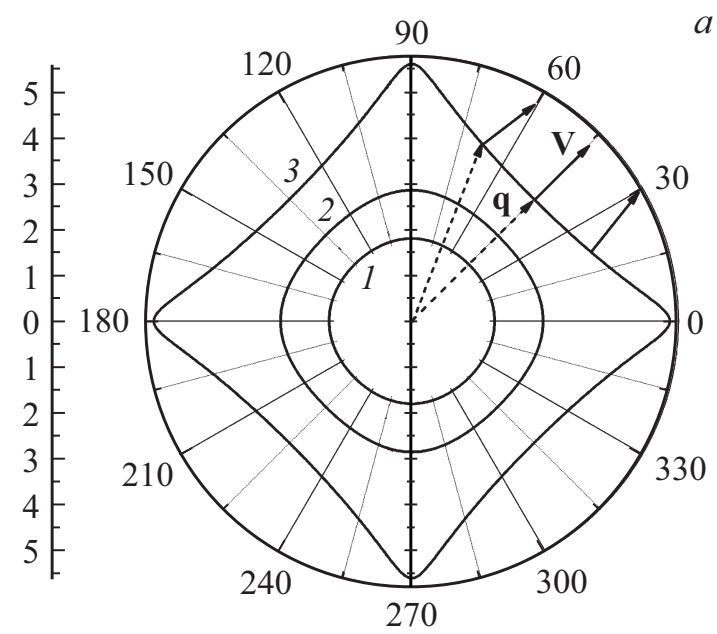

$a$

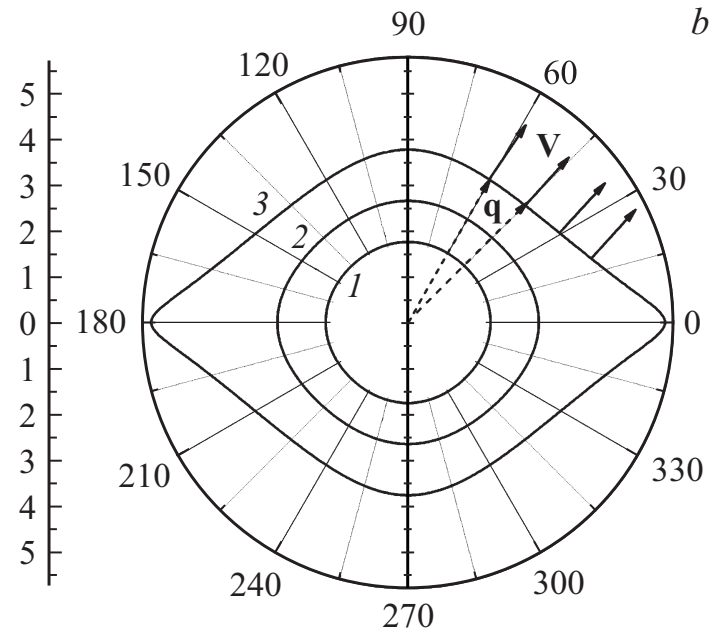

Рис. 2. Сечения поверхностей постоянной частоты $a q(\theta)$ в ГЦК-решетке плоскостями $\{100\}(a)$ и $\{110\}(b)$ в модели, учитывающей обменное взаимодействие с первыми и вторыми соседями с параметром $\xi=0.8$ для частот $\omega=5 \omega_{E}$ (кривые 1 ), $10 \omega_{E}$ (кривые 2) и $15.9 \omega_{E}$ (кривые 3 ).
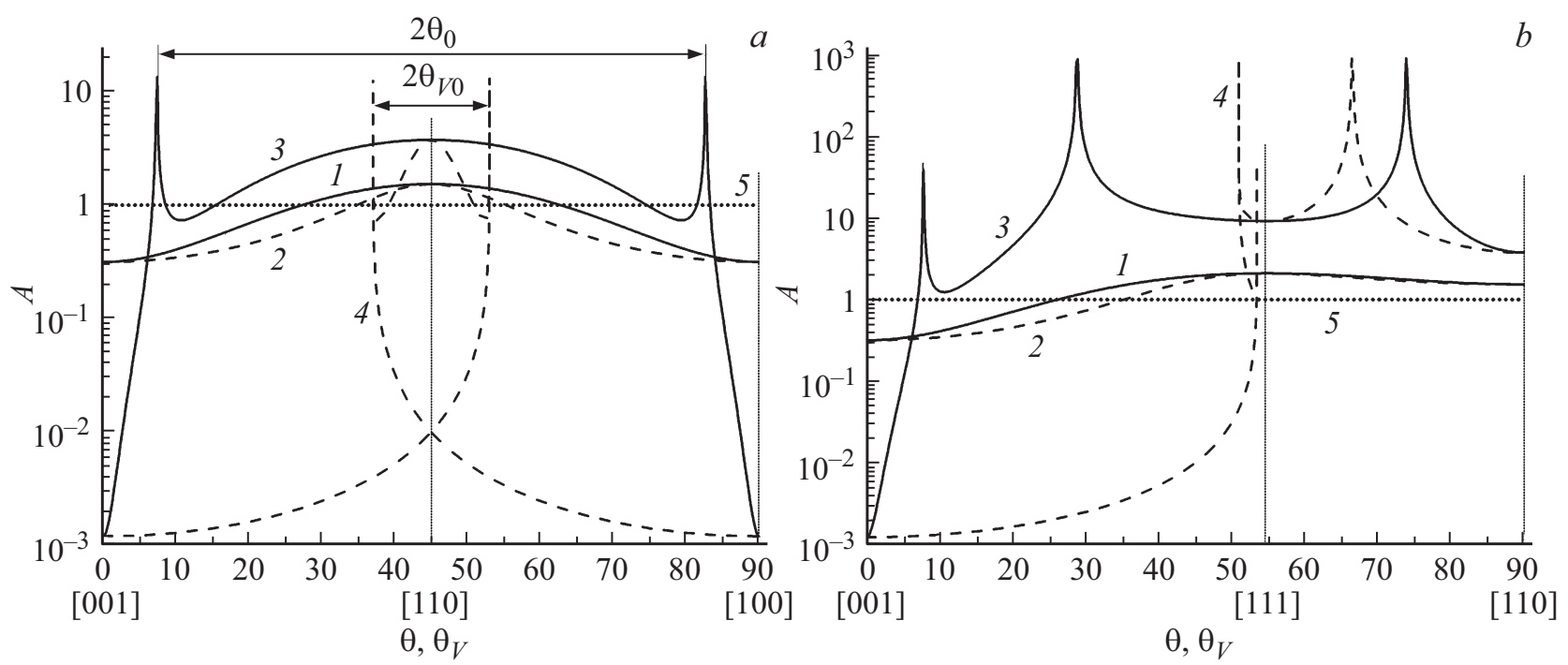

Рис. 3. Угловые зависимости коэффициента усиления $A(\theta)$ (кривые $1,3,5)$ и $A\left(\theta_{V}\right)$ (кривые 2,4$)$ в плоскости грани куба $(a)$ и в диагональной плоскости $(b)$ для параметра $\xi=0.8$ для частот спиновых волн $\omega=10 \omega_{E}$ (кривые 1,2$)$ и $\omega=15.9 \omega_{E}$ (кривые 3,4 ). Линии 5 - изотропный случай $(a q \ll 1)$. Углы $\theta_{0}$ и $\theta_{V 0}$ указывают на особые точки коэффициента усиления.

фокусировки функция $\theta_{V}(\theta)$ несимметрична, и для соответствующего анализа необходимо вводить дополнительные углы. Анализ функции $\theta_{V}(\theta)$ для этого случая показал, что ее локальные экстремумы соответствуют положениям расходимости коэффициента усиления $A(\theta)$ в q-пространстве при $\theta=7.8^{\circ}$ и $29.0^{\circ}$ (см. рис. 3). В координатном представлении $(\mathbf{V}$-пространстве) пики коэффициента усиления находятся вблизи направления [111] при $\theta_{V}=50.9^{\circ}$ и $53.4^{\circ}$, поскольку в этом случае изоэнергетическая поверхность почти плоская, и угол $\theta_{V 0} \approx 1^{\circ}$.

С изменением знака параметра $\xi$ происходит изменение направлений фокусировки. При $\xi=-0.5$ (этот случай относится к кристаллу $\mathrm{EuS}$ ) на частотах $\omega=4 \omega_{E}$ в окрестности направления [110] изоэнергетическая поверхность имеет плоский участок, что приводит к сильной фокусировке спиновых волн и расходимости коэффициента усиления (см. рис. 4 и табл. 2). В направлении [100] имеет место локальная фокусировка, а в направлении [111] - дефокусировка спиновых волн. Увеличение частоты спиновых волн до $\omega \approx 5.9 \omega_{E}$ приводит к качественному изменению картины фокусировки: в направлении [110] коэффициент усиления меньше единицы $(A=0.54)$, а в окрестности этого направления в диагональной плоскости формируется каустика магнонов. В направлении [100] наблюдается фокусировка и каустика спиновых волн в окрестности этого направления. В направлении [111], как и в предыдущем случае, происходит дефокусировка спиновых волн (см. рис. 1, $b$ и 4 ). 

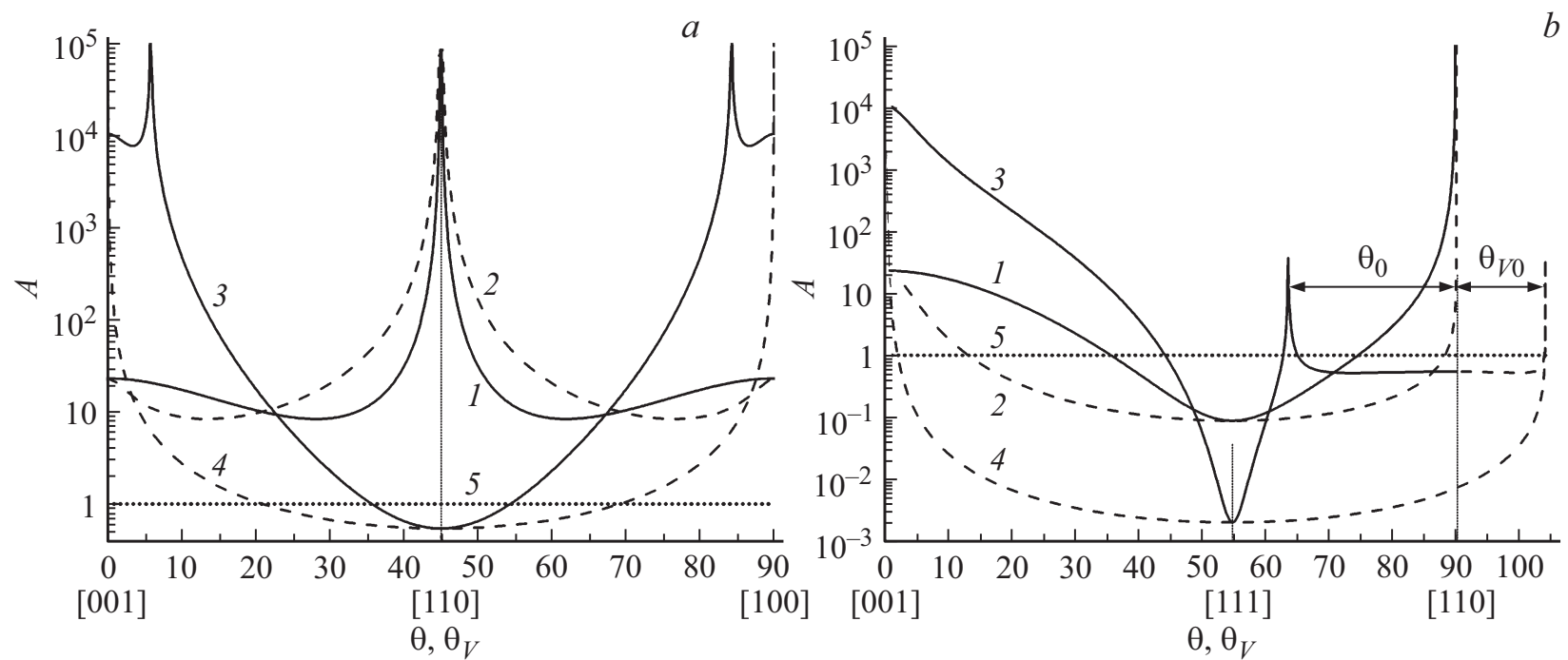

Рис. 4. Угловые зависимости коэффициента усиления $A(\theta)$ (кривые $1,3,5)$ и $A\left(\theta_{V}\right)$ (кривые 2,4$)$ в плоскости грани куба $(a)$ и в диагональной плоскости $(b)$ для параметра $\xi=-0.5$ для частот спиновых волн $\omega=4 \omega_{E}$ (кривые 1,2$)$ и $\omega=5.9 \omega_{E}($ кривые 3,4$)$. Линии 5 - изотропный случай $(a q \ll 1)$. Углы $\theta_{0}$ и $\theta_{V 0}$ указывают на особые точки коэффициента усиления.
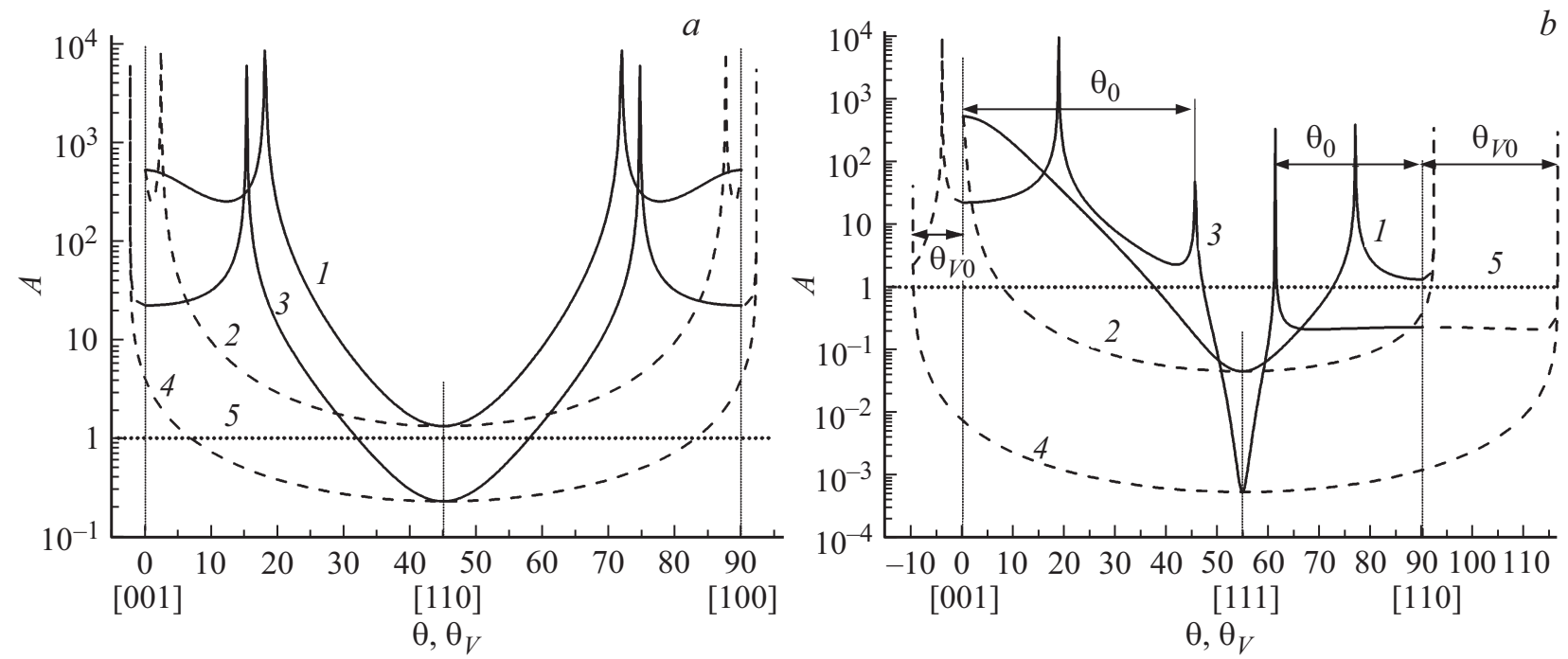

Рис. 5. Угловые зависимости коэффициента усиления $A(\theta)$ (кривые $1,3,5)$ и $A\left(\theta_{V}\right)$ (кривые 2,4$)$ в плоскости грани куба $(a)$ и в диагональной плоскости $(b)$ для параметра $\xi=-0.8$ для частот спиновых волн $\omega=\omega_{E}$ (кривые 1,2 ) и $\omega=2.3 \omega_{E}$ (кривые 3,4 ). Линии 5 - изотропный случай $(a q \ll 1)$. Углы $\theta_{0}$ и $\theta_{V 0}$ указывают на особые точки коэффициента усиления.

Дальнейшее уменьшение параметра $\xi$ до -0.8 приводит к формированию новых точек каустики в диагональной плоскости. Так, на частотах $\omega=2.3 \omega_{E}$ в этой плоскости коэффициент усиления в q-пространстве имеет особенность в окрестности трех симметричных направлений: [100], [110] и [111]. В координатном представлении (V-пространстве) эти пики переходят к направлениям типа [100] и [111], а в направлении [111] имеет место дефокусировка магнонов с коэффициентом усиления $A=5.5 \cdot 10^{-4}$ (см. рис. $5, b$ ). В плоскости грани куба, как и в случае $\xi=-0.5$, формирование каустики происходит в окрестности направления [100] (см. рис. 5,a).
Предложенный метод анализа фокусировки спиновых волн позволил определить угловые распределения средних плотностей магнонных состояний для областей фокусировки и дефокусировки магнонов и оценить величины этих плотностей в плоскости грани куба и диагональной плоскости для значений параметра $\xi=0.8$, -0.5 и -0.8 (см. табл. 1). Чем более плоский участок на изоэнергетической поверхности, тем больше плотность состояний $n_{F I}$.

Подводя итог, заметим, что фокусировка и каустика спиновых волн формируются для волновых векторов во второй половине зоны Бриллюэна, причем значения параметра $\xi$ частоты, при которых реализуется этот эф- 
фект, различны. Так, для $\xi=0$ и 0.8 фокусировка магнонов имеет место при частотах $\omega \gtrsim 10 \omega_{E}$; для $\xi=0.2-$ при $\omega \gtrsim 13 \omega_{E}$; для $\xi=-0.5-$ при $\omega \gtrsim 4 \omega_{E}$; для $\xi=-0.8-$ при $\omega \gtrsim \omega_{E}$. Случаи $\xi=0.2$ и -0.5 относятся к кристаллам $\mathrm{EuO}$ и EuS, соответственно. Для них известны частоты $v_{E}=\omega_{E} / 2 \pi=2 S J_{1} / 2 \pi \hbar=91.9 \mathrm{GHz}$ для $\mathrm{EuO}$ и $29.2 \mathrm{GHz}$ для EuS. Согласно приведенным выше оценкам фокусировка и каустика магнонов в этих соединениях будут формироваться при частотах $v \gtrsim 1.2 \mathrm{GHz}$ для $\mathrm{EuO}$ и $v \gtrsim 0.12 \mathrm{GHz}$ для EuS.

\section{5. Выводы}

В настоящей работе нами проанализированы особенности процесса передачи энергии из точечного источника спиновых волн в магнитных полупроводниках на примере двух базовых соединений $\mathrm{EuO}$ и $\mathrm{EuS}$, имеющих структуру каменной соли (ГЦК). Для каждого типа решетки был определен интервал, где реализуются условия фокусировки спиновых волн. Установлены направления в кристалле, а также необходимые условия для формирования каустики - огибающей семейства магнонов (эволюты), не сходящихся в одной точке, вблизи которой резко возрастает интенсивность магнонного поля. Проанализировано также изменение условий фокусировки магнонов при изменении знака параметра обменного взаимодействия (обменного интеграла) со вторыми соседями. Показано, что в длинноволновом приближении фокусировка обменных спиновых волн отсутствует: она наблюдается лишь для волновых векторов во второй половине зоны Бриллюэна $(a q \gtrsim \pi)$. Для ее реализации не требуется внешнего магнитного поля и размерных эффектов.

Основные результаты работы можно сформулировать следующим образом:

1. Фокусировка и каустика магнонов будут формироваться на частотах $v \gtrsim 1.2 \mathrm{GHz}$ для ЕuО и $v \gtrsim 0.12 \mathrm{GHz}$ для EuS.

2. Определены направления фокусировки, дефокусировки и каустики магнонов. В кристалле $\mathrm{EuO}$ фокусировка имеет место в направлениях [110] и [111], а дефокусировка - в направлениях типа [100]. В кристаллах $\mathrm{EuS}$ картина фокусировки качественно отличается: фокусировка магнонов наблюдается в направлении [100], в направлении [111] - дефокусировка, а в окрестности направления [110] формируется каустика магнонов (значения углов $\theta_{V 0}$, определяющих положения каустик, представлены в табл. 1).

3. Изменение величины и знака параметра обменного взаимодействия со вторыми соседями $\xi$ приводит к изменению условий фокусировки магнонов. Так, например, для $\xi=0.8$ в направлении [100] происходит дефокусировка, а для $\xi=-0.8-$ фокусировка магнонов. В направлениях [110] и [111] для случая $\xi=0.8$ имеет место фокусировка и формирование каустики магнонов в окрестности этих направлений, а для случая $\xi=-0.8-$ дефокусировка спиновых волн (см. табл. 1 и 2).

\section{Список литературы}

[1] Э.Л. Нагаев. УФН 117, 3, 437 (1975).

[2] S.G. Altendorf, A. Reisner, B. Tam, F. Meneghin, S. Wirth, L.H. Tjeng. Phys. Rev. B 97, 165422 (2018).

[3] A.S. Borukhovich, N.I. Ignat'eva, K.I. Yanushkevich, A.I. Stognii, Yu.A. Fedotova. JETP Lett. 89, 4, 191 (2009).

[4] A.S. Borukhovich, A.V. Troshin. Creation and Research of Properties of Multilayers and Superconducting Tunnel Transitions with the Participation of EuO. Springer International Publishing, Cham (2018). 121-138 p.

[5] A.S. Borukhovich, A.V. Troshin. Features of Formation of Electron Band Structure and Physical Properties of Ferromagnetic Semiconductor EuO. Springer International Publishing, Cham (2018). 3-35 p.

[6] O.W. Dietrich, A.J. Henderson, H. Meyer. Phys. Rev. B 12, 2844 (1975).

[7] L. Passell, O.W. Dietrich, J. Als-Nielsen. Phys. Rev. B 14, 4897 (1976).

[8] T. Kasuya. IBM J. Res. Dev. 14, 3, 214 (1970).

[9] H.J. Maris. J. Acoust. Soc. Am. 50, 812 (1971).

[10] A.G. Every. Phys. Rev. B 24, 3456 (1981).

[11] Cz. Jasiukiewicz, T. Paszkiewicz, D. Lehmann. Z. Phys. B Condens. Matter 96, 2, 213 (1994).

[12] J. Philip, K.S. Viswanathan. Phys. Rev. B 17, 12, 4969 (1978).

[13] M. Lax, V. Narayanamurti. Phys. Rev. B 22, 4876 (1980).

[14] G.A. Northrop, J.P. Wolfe. Phys. Rev. B 22, 6196 (1980).

[15] J.P. Wolfe. Imaging Phonons Acoustic Wave Propagation in Solids. Cambridge University Press, N.Y. (1998). 411 p.

[16] И.Г. Кулеев, С.М. Бахарев. ФТТ 60, 7, 1260 (2018).

[17] И.Г. Кулеев, И.И. Кулеев, С.М. Бахарев, В.В. Устинов. Фокусировка фононов и фононный транспорт в монокристаллических наноструктурах. УМЦ УПИ, Екатеринбург (2018). $256 \mathrm{c}$.

[18] I.I. Kuleyev, S.M. Bakharev, I.G. Kuleyev, V.V. Ustinov. Phys. Status Solidi C 14, 1600263 (2017).

[19] И.Г. Кулеев, И.И. Кулеев, С.М. Бахарев, В.В. Устинов. Физика металлов и металловедение 118, 1, 12 (2017).

[20] Д. Илуэлл, И. Остин. УФН 106, 337 (1972).

[21] B. Taylor, H.J. Maris, C. Elbaum. Phys. Rev. Lett. 23, 416 (1969).

[22] А.В. Погорелов. Дифференциальная геометрия. Наука, М. (1974). $176 \mathrm{c.}$

[23] В.И. Арнольд. Теория катастроф. Наука, М. (1990). 128 с.

Редактор Т.Н. Василевская 